\title{
RAPID INVER'TING OF THE POLARIZATION OF A NEUTRON BEAM USING LARGE AMPLITUDE OSCILLATING MAGNETIC FIELDS
}

\author{
H. KENDRICK* and J. S. KING \\ Nuclear Engineering Department, The University of Michigan, Ann Arbor, Michigan, U.S.A. \\ S. A. WERNER \\ Scientific Research Staff, Ford Motor Company, Dearborn, Michigan, U.S.A. \\ A. ARROTT \\ Scientific Research Staff, Ford Motor Company, Dearborn, Michigan, U.S.A. \\ and \\ Nuclear Engineering Department, The University of Michigan, Ann Arbor, Michigan, U.S.A. \\ and \\ Department of Physics, Simon Fraser University, Burnaby, British Columbia
}

Received 19 August 1969

During the course of designing and constructing a pulsedneutron-polarization-inverter (PNPI) to be used in the study of magnetic excitations in solids, it was necessary to re-examine the oscillating field method of magnetic resonance in the region where the amplitude of the rf field $B_{1}$ is comparable to the dc field $B_{0}$. It is found that the phase of the rf field at the instant the

\section{Introduction}

Following a suggestion of $\mathbf{R}$. Nathans, we have been designing and implementing an apparatus for rapidly inverting the polarization of a neutron beam. In order to study magnons it is useful to have pulses of neutrons of one polarization for 10 to $40 \mu$ sec. In the course of this work we have re-examined the oscillating field method of magnetic resonance. As distinct from the usual applications ${ }^{1}$ ) where the resonance frequency and line widths are the important parameters, for the pulsed-neutron-polarization-inverter (PNPI) the degree to which the moments are reversed and the width (in time) of the polarization inversion pulses are the main considerations $^{2}$ ). To have well-defined pulses it is necessary that the region of space over which this high frequency magnetic field acts on the neutron polarization be sharply limited. To meet this condition we have used an oscillating of field with an amplitude comparable to the dc magnetic field. A description of the resonance under these conditions was not found in the literature.

The motion of a magnetic moment in a de field with a rotating $\mathrm{rf}$ field applied perpendicularly is the usual starting point for discussions of magnetic reso-

* Present address: Gulf General Atomic, San Diego, California, U.S.A. neutron enters the spatial region of the field becomes an increasingly important consideration as $B_{1} / B_{0}$ becomes comparable to 1 . Analog and digital computer solutions are given for the equations of motion of the neutron's magnetic moment in the presence of various field configurations important in the construction of a PNPI.

nance. It is then pointed out that oscillating field problems "can usually be treated as one involving a rotating field since an oscillatory field is equivalent to two opposite rotating fields and only the component rotating in the direction to be able to give a resonance... has an important effect in most problems" 3 ). There is then given a reference to Bloch and Siegert ${ }^{4}$ ) and to Stevenson ${ }^{5}$ ) who showed that there is a very slight (usually experimentally negligible) shift in the resonance frequency when one considers the oscillating field problem ${ }^{6}$ ). But it seems that the oscillating field problem has not been solved. As we had some rather specific questions to answer through solutions of the equation of motion for a magnetic moment in an oscillating field of large amplitude we programmed them first for an analog computer to get a good idea of the nature of the motion and then for a digital computer to find answers where considerable precision was necessary. We present results of these computer studies because of their application to the pulsedneutron-polarization-inverter (PNPI) and also because of an academic interest in this unsolved problem in magnetic resonance.

The particular problem we have in mind is that of a well-collimated, well-polarized beam of almost monoenergetic thermal neutrons (velocity $\approx 4 \times 10^{3} \mathrm{~m} / \mathrm{sec}$ ) 
in a $\mathrm{dc}$ field of $\approx 150 \mathrm{G}$. The beam enters a single layer coil of square cross section and for a distance of $2.8 \mathrm{~cm}$ is subject to an oscillating magnetic field of $\approx 50 \mathrm{G}$ at $\approx 0.5 \mathrm{MHz}$. The neutron is subjected to the rf magnetic field only while it is inside the coil, for the coil has its axis perpendicular to the flight path as well as perpendicular to the vertical de magnetic field. That is, the neutrons go through the aluminum wire of the coil and its aluminum oxide insulation. (This coil arrangement is in contrast to previous polarized neutron work in which the coil axis is along the beam.) The polarization of the neutrons is inverted by adjusting the de field to make the Larmor precession frequency of the neutrons match the frequency of the oscillating field, and also by adjusting the amplitude of the rf field so that the correct value is obtained for the integral of the rf field over the time to which the neutron is subjected to it. Because our choice of frequency of the rf committed us to flipping the neutron magnetic moment in a small number of cycles we wondered how the completeness of the polarization inversion would be affected by the fact that each neutron would enter the coil at a different phase of the oscillating field. As the coil is of finite length we were also concerned with the effect of the nonuniformity of the rf field, and because of the way in which we apply the dc field we were also concerned with the effects of nonuniformity in it.

The problem as described above is for a steady turning over of all the neutrons. Nothing has been said about pulses. The pulsing is accomplished by using a second coil surrounding the rf coil but with its axis vertical. The neutrons go through the wire of this coil as well as that of the rf coil. This we call the dc coil and it too is a single layer of aluminum wire. The purpose of the dc coil is to reduce the dc magnetic field which is supplied by iron plates and alnico magnets. The resonance condition is maintained as long as there is no current in the dc coil. The neutrons are all flipped as they go through the rf coil until a pulse of current is supplied to the dc coil. This destroys the resonance condition and the neutrons pass through unflipped for the duration of the pulse. Our principal concern in this paper is to study the flipping process in the absence of the pulse in the dc coil. But by applying steady currents to the dc coil we are able to use the PNPI apparatus to compare our conclusions from the computer calculations with experiment.

In presenting the results for the oscillating field case it is necessary for clarity to first review the rotating field resonance equations of motion. We then treat the oscillating field case. We give the integral equations to be solved by computer, present and discuss the com- puter results, and make some comparisons with experient. We also add a few words on the performance of the PNPI.

\section{Rotating field in the region of a constant field}

The discussion by Rabi, Ramsey and Schwinger ${ }^{3}$ ) shows the extensive applicability of the classical treatment in which the equation of motion in a stationary coordinate system is

$$
\mathrm{d} \boldsymbol{M} / \mathrm{d} t=\gamma \boldsymbol{M} \times \boldsymbol{B},
$$

where $\boldsymbol{M}$ is the magnetic moment, $\gamma$ the gyromagnetic ratio, and $\boldsymbol{B}$ the magnetic induction. The magnetic moment is $\gamma \hbar\langle S\rangle$ where $\langle S\rangle$ is the expectation value of the neutron spin. In a frame of reference rotating with angular velocity $\omega$ (the sense being determined by the right-hand screw rule) the equation of motion is

$$
\begin{aligned}
\partial \boldsymbol{M} / \partial t & =\gamma \boldsymbol{M} \times(\boldsymbol{B}+\boldsymbol{\omega} / \gamma) \\
& =\gamma \boldsymbol{M} \times \boldsymbol{B}_{\mathrm{er}},
\end{aligned}
$$

where $\boldsymbol{B}_{\mathrm{er}}$ is the effective field in the rotating coordinate system. For the rotating coordinate system the dc field is along $z$-axis and the $x$-axis is chosen to coincide with the direction of the rf field. To produce complete resonance one wants $\boldsymbol{B}_{\mathrm{er}}$ to have a component only along the $x$-direction so that the moment precesses in the $z y$ plane. Then this condition is maintained just long enough for the precession to go through $\left(n+\frac{1}{2}\right)$ cycles. To reduce $\boldsymbol{B}_{e r}$ to a single component along the $x$-axis the direction of rotation is to be that of the left hand screw or

$$
\omega=-\omega k
$$

where $\boldsymbol{k}$ is chosen so that

$$
\boldsymbol{B}_{0}=B_{0} \boldsymbol{k}(=\mathrm{dc} \text { field }) .
$$

The rotating coordinate system is completed by recalling that

Thus

$$
\boldsymbol{B}_{1}=B_{1} \boldsymbol{i}(=\text { rotating field amplitude) }
$$

$$
\boldsymbol{B}_{\mathrm{er}}=B_{1} \boldsymbol{i}+\left(B_{0}-\omega / \gamma\right) \boldsymbol{k},
$$

and the precession about $\boldsymbol{B}_{\mathrm{er}}$ gives as the solutions to eq. (2)

$$
\begin{aligned}
& M_{x}(t)=M_{0} \omega_{1}\left(\omega_{0}-\omega\right)[1-\cos a t] / a^{2}, \\
& M_{y}(t)=M_{0} \omega_{1}[\sin a t] / a, \\
& M_{z}(t)=M_{0}\left[\left(\omega_{0}-\omega\right)^{2}+\omega_{1}^{2} \cos a t\right] / a^{2},
\end{aligned}
$$

where the initial conditions $M_{x}(0)=M_{y}(0)=0$ and 
$M_{z}(0)=M_{0}$ have been used. The symbols $a, \omega_{1}$ and $\omega_{0}$ are defined by

and

$$
\begin{aligned}
& \omega_{0}=\gamma B_{0}, \\
& \omega_{1}=\gamma B_{1},
\end{aligned}
$$

$$
a^{2}=\left(\omega_{0}-\omega\right)^{2}+\omega_{1}^{2} \text {. }
$$

These solutions are sketchud in fig. 1 . The oscillations in $\boldsymbol{M}(t)$ are large only when $\omega_{0}$ approaches $\omega$. And at resonance $\boldsymbol{M}$ simply rotates in the $y z$ plane. The flipping probability $P(t)$ is related to $M_{z}(t)$ by

$$
\begin{aligned}
P(t) & =\frac{1}{2}\left[1-M_{z}(t)\right] \\
& =\left[1+\left(\Delta \omega / \omega_{1}\right)^{2}\right]^{-1} \sin ^{2}\left\{\left[1+\left(\Delta \omega / \omega_{1}\right)^{2}\right]^{\frac{1}{2}} \omega_{1} t / 2\right\},
\end{aligned}
$$

where $\Delta \omega=\omega_{0}-\omega$.

At resonance

$$
P(t)=\sin ^{2} \frac{1}{2} \omega_{1} t
$$

and complete flipping occurs for

$$
t=\left(\pi / \omega_{1}\right)(2 n+1) \equiv T_{1}(2 n+1) .
$$

The full-width-at-half-maximum of the resonance is
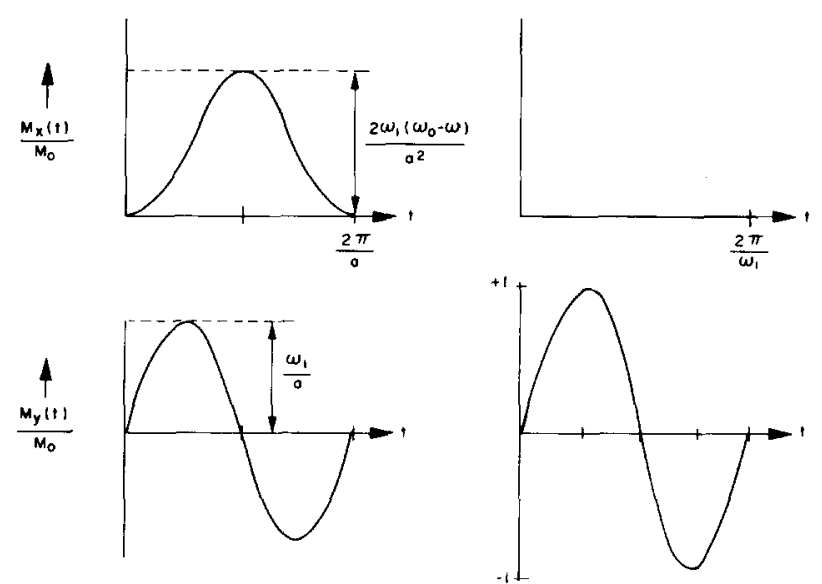

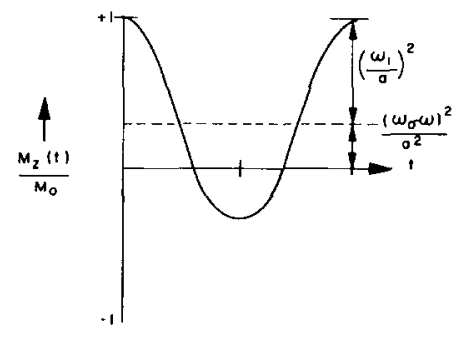

(a)

Off Resonance

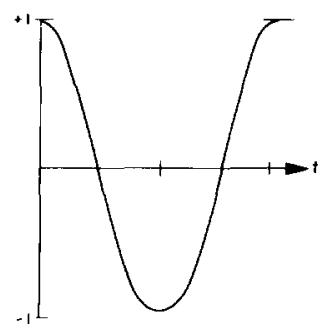

(b)

At Resonance
Fig. 1. Time dependence of the components of magnetization in a coordinate system rotating with the perpendicular magnetic field: (a) off resonance, (b) at resonance.
$\Delta \omega=2 \omega_{1}$. If at the resonant frequency one considers changes in the amplitude of the rf such that the component of the rf field along the $y$-axis in the rotating coordinate system remains very small compared to the component along the $x$-axis, the precession remains essentially in the $y-z$ plane. Under those conditions the only effect of time variation of the amplitude of the rotating $\mathrm{rf}$ is on the time dependence of the phase of the precession in the $y-z$ plane. The precession will still go through the point where $M_{y}$ is zero and $M_{z}$ is reversed. The resonance condition on the rf field is that

$$
\int_{0}^{T} \omega_{1}(t) \mathrm{d} t=\pi(2 n+1) .
$$

The problem with an oscillating field is that the precession is not restricted to the $y-z$ plane. Hence it is not yet clear that the magnetization components $M_{x}$ and $M_{y}$ can be made zero simultaneously. It turns out that they can be by finding a new resonance frequency and a new resonance amplitude. This can be seen by looking at the equation of motion in the $x-y$ plane. The variables $\omega$ and $\omega_{1}$ determine the path. There is a one to one correspondence between the $M_{x}$ and $M_{y}$ coordinates at $t=T$ and the choice of $\omega$ and $\omega_{1}$. But this correspondence may depend upon the phase of the oscillating of when the neutron enters the coil. Thus while it is clear that the conditions for complete flipping can be found for any one choice of this phase, it is likely that for a different phase one would need different conditions. These sage comments were formulated only after we had investigated the oscillating field problem by the computer experiments described below.

\section{Oscillating field in the region of a constant field}

\subsection{Preliminary COMMENTS}

In a rotating coordinate system the rotating field problem reduces to the precession of the magnetic moment about a static field, $\boldsymbol{B}_{\mathrm{er}}$. In a rotating coordinate system the oscillating field problem has in addition to a static field

$$
\boldsymbol{B}_{\mathrm{ers}}=\frac{1}{2} \boldsymbol{B}_{1} \boldsymbol{i}+\left(B_{0}-\omega / \gamma\right) \boldsymbol{k}
$$

a rotating field of angular frequency $2 \omega$

$\boldsymbol{B}_{\mathrm{err}}=\frac{1}{2} B_{1}[\boldsymbol{i} \cos 2(\omega t+\varphi)+\boldsymbol{j} \sin 2(\omega t+\varphi)]$.

We have here taken $B_{1}$ as the amplitude of the $\mathrm{rf}$ oscillating field. (That is, $\frac{1}{2} B_{1}$ is the amplitude of each of the two counter rotating fields.)

The substitution of the sum of these two fields into the equation of motion yields a set of differential 
equations which cannot be solved analytically. Physically, however, we expect the oscillating field solutions to look like the rotating field solutions except for a modulation at the angular frequency $2 \omega$. The case for $B_{0} \gg B_{1}$ has been considered by Bloch and Siegert and Stevenson. The approximate result is that

$$
\omega_{\mathbf{r}} / \omega_{0}=1+\omega_{1}^{2} / 4 \omega_{0}^{2}
$$

where $\omega_{\mathbf{r}}$ is the resonance frequency when the effective field is composed of the static field [eq. (12)] plus the field oscillating at frequency $2 \omega$ [eq. (13)]. We define as before,

and

$$
\omega_{1}=\frac{1}{2} \gamma B_{1}
$$

$$
\omega_{0}=\gamma B_{0} .
$$

When the relative field magnitudes $B_{1}$ and $B_{0}$ are such that $B_{0} \gg B_{1}$ this modulation is of little importance. But when $B_{0} \approx B_{1}$ the modulations should be about equal to the response and one may well wonder what happens to the degree to which the neutrons entering at different phases can have their spins reversed. One advantageous effect of large rf fields can be seen from the equations for the rotating case. The larger the $\mathrm{rf}$ field, the broader the resonance, and the less the demands on the stability of the apparatus for maintaining a maximum probability for flipping neutrons over long periods of time. One therefore hopes that the phase problem is not serious.

\subsection{ANALOG COMPUTER EXPERIMENTS}

A preliminary look at the problem for $B_{1}$ comparable to $B_{0}$ was obtained using an analog computer inasmuch as it is particularly suited to the solution of oscillatory problems. In integral form the equations of motion for the oscillating field in a stationary frame are:

$$
\begin{aligned}
M_{x}(t)= & M_{x}(0)+\int_{0}^{t} \omega_{0} M_{y}\left(t^{\prime}\right) \mathrm{d} t^{\prime}, \\
M_{y}(t)= & M_{y}(0)+\int_{0}^{t} 2 \omega_{1} M_{z}\left(t^{\prime}\right) \cos \left(\omega t^{\prime}+\varphi\right) \mathrm{d} t^{\prime}- \\
& -\int_{0}^{t} \omega_{0} M_{x}\left(t^{\prime}\right) \mathrm{d} t, \\
M_{z}(t)= & M_{z}(0)-\int_{0}^{t} 2 \omega_{1} M_{y}\left(t^{\prime}\right) \cos \left(\omega t^{\prime}+\varphi\right) \mathrm{d} t^{\prime} .
\end{aligned}
$$

The equations apply for any arbitrary time dependence of the two fields

and

$$
B_{1}=2 \omega_{1} / \gamma
$$

$$
B_{0}=\omega_{0} / \gamma \text {. }
$$

Figs. $2 \mathrm{a}, 2 \mathrm{~b}$ and $2 \mathrm{c}$ show the time dependence of the three components of $\boldsymbol{M}$ for increasing rf amplitude at the rotating field resonance condition $\omega=\omega_{0}$. Note that even at the small relative amplitude $\left(B_{1} / B_{0}=\right.$ $\left.2 \omega_{1} / \omega_{0} \ll 1\right)$ small ripples appear in the components of $M$. As $B_{1} / B_{0}$ increases these ripples begin to increase and at $B_{1} / B_{0}=1.0$ the effect of the extra rotating component of the oscillating field becomes very pronounced. The extra rotating component of the field actually aids in the rate at which the spin is turning over for half of its cycle, and then slows down the rate at which it is turning over for the other half of the cycle.

If one disregards the modulation, the curves in fig. 2 for the $M_{z}$ component are just what one expects for the rotating field case. Despite the lack of accuracy in these results. One can see that the oscillating field method was not quite perfect. For the $M_{x}$ and $M_{y}$ components do not go to zero when the $M_{z}$ component appears to go to complete reversal. This is particularly evident when the choice of initial phase is varied as is seen in figs. $3 a, b$ and c. Fig. $3 a$ is a repeat of the experiment on the left hand side of fig. $2 \mathrm{c}$ but on an extended time scale. Note that at the end of two cycles $M_{y}$ goes to zero and $M_{z}$ goes to $\approx 1$ but $M_{x}$ does not go to zero. Yet $M_{x}$ is at a minimum. If the phase is shifted by $\pi / 2$, $M_{x}$ goes through zero after two complete cycles but now $M_{y}$ is at a local maximum and $M_{z}$ is less than 1 for both slightly earlier and slightly later times. Examination of the curves for $\varphi=\frac{1}{4} \pi$ shows that $M_{z}$ is not symmetric in time and consequently a better flipping probability is found for times slightly longer than two cycles.

While the analog computer calculations were sufficient to assure us that there were no serious problems to be encountered in using large amplitude oscillating fields for flipping a beam of neutrons, we were curious to see more precisely how the flipping conditions were changed from the rotating field case. This led to a set of digital computer experiments.

\subsection{Digital COMPUTER EXPERIMENTS}

The first object of these experiments was to find the resonance condition for perfect flipping of a neutron in the time given by its transit through the rf field. The neutron wave length was taken as $1.05 \AA$, the path through the oscillating rf field was $2.80 \mathrm{~cm}$, and the frequency was chosen at $530 \mathrm{kHz}$. The equations of motion were rewritten as finite difference equations. To find the resonance conditions both $B_{1}$ and $\omega$ were varied to make the flipping probability as close to unity as possible. An inspection of the rotating field equations [eq. (6)] shows how to go about this. One notices that near complete flipping the $M_{x}$ component goes 
H. KENDRICK et al.

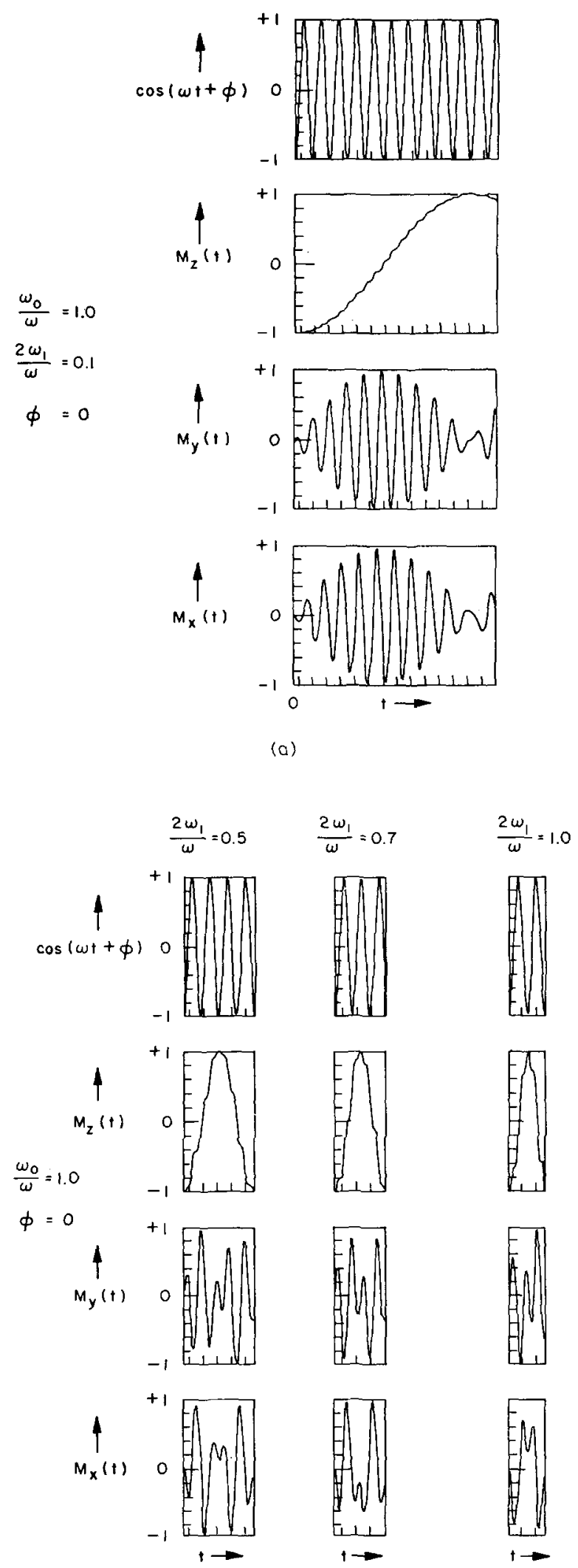

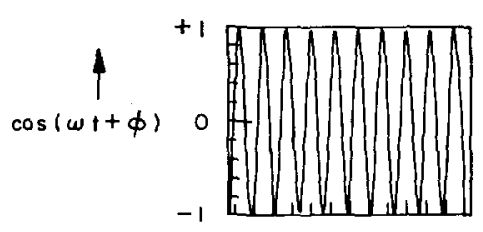

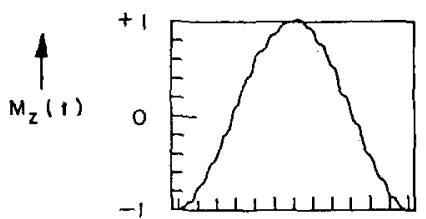

$\frac{\omega_{0}}{\omega}=1.0$

$\frac{2 \omega_{1}}{\omega}=0.2$

$\phi=0$
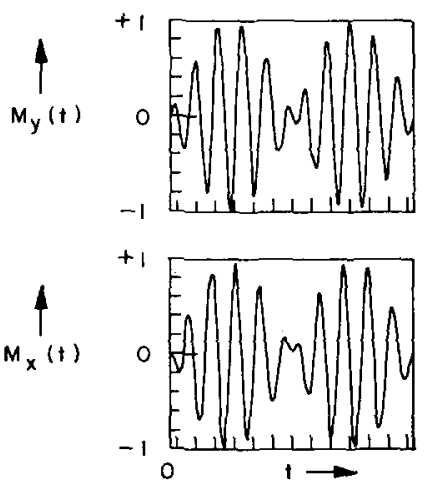

(b)

Fig. 2. The time dependence of the components of magnetization in a stationary coordinate system for the perpendicular magnetic field oscillating at the frequency corresponding to the frequency for resonance with a rotating field. The neutron enters a region of constant oscillating field when the field is at a maximum. The amplitude is increased starting with $B_{1}=0.1 B_{0}$ in $2 \mathrm{a}$. $B_{1}=0.2$ in $2 \mathrm{~b}$ and $B_{1}=0.5 B_{0}, 0.7 B_{0}$ and $B_{0}$ in $2 \mathrm{c}$. 

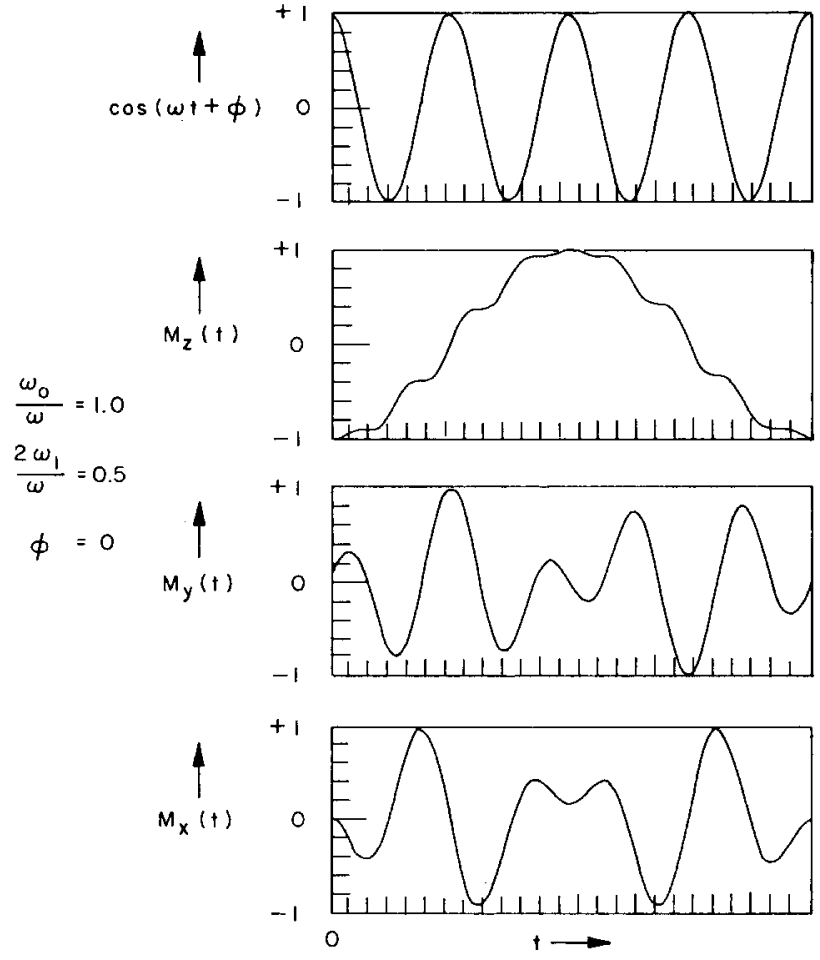

(a)

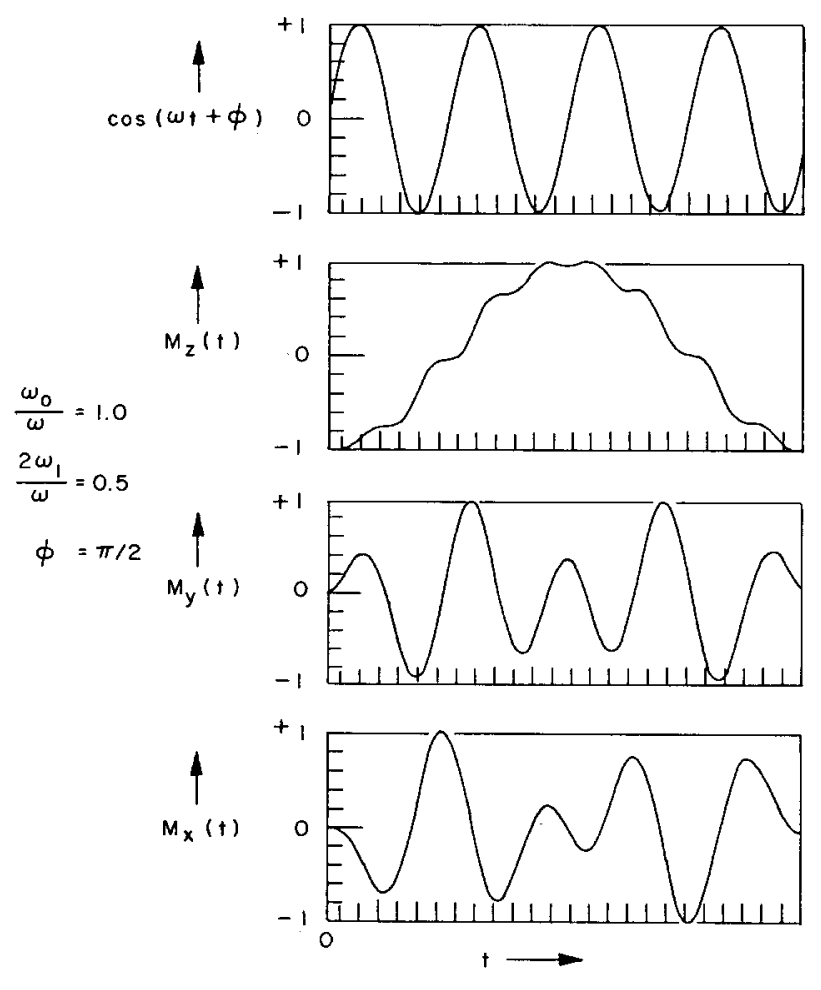

(c)
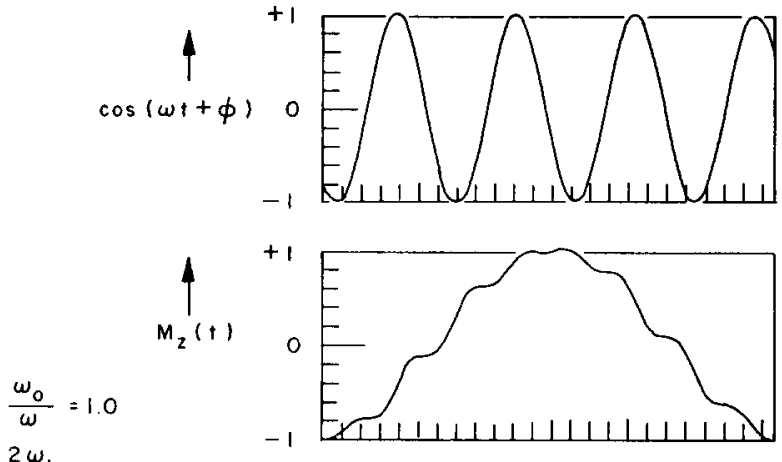

$\frac{2 \omega_{1}}{\omega}=0.5$

$\phi=\pi /\left.4\right|_{M_{y}(1)}$
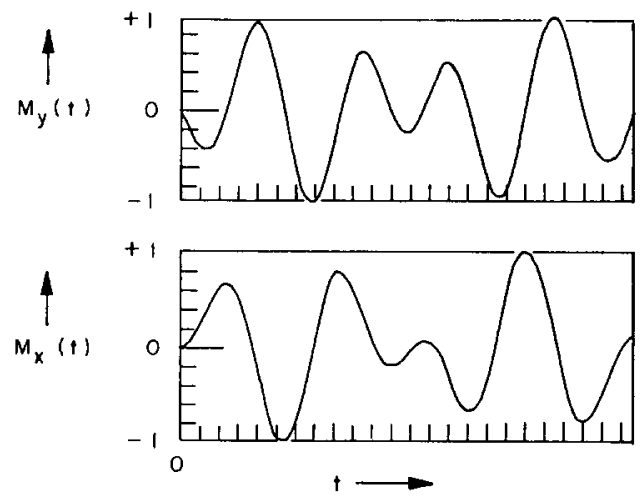

(b)

Fig. 3. The effect of the phase of the oscillating field is shown for $B_{1}=0.5 B_{0}$. In $3 a$ the neutron enters in phase.

In $3 b$ the phase is $\pi / 4$ and in $3 c$ the phase is $\pi / 2$. 
linearly through zero as $\omega$ passes $\omega_{0}$, and similarly the $M_{y}$ component goes linearly through zero as $\omega_{1}$ passes $\pi / \tau$, where $\tau$ is the transit time of the neutrons. For the oscillating field case the computer results also show a linear variation of $M_{x}$ at $t=\tau$ with frequency for fixed $B_{1}$ and a linear variation of $M_{y}$ with $B_{1}$ for fixed frequency. From the crossover points one obtains an improved value of $B_{1}$ and $\omega$ for complete flipping at $t=\tau$ and the iteration of this procedure converges very rapidly. The iteration was terminated when $M_{z}(\tau)$ reached -0.9999 with the values

and

$$
\omega=\omega_{0} / 0.996045
$$

$$
\omega_{1}=0.991225 \omega_{\mathrm{R}},
$$

where $\omega_{\mathrm{R}}$ is the amplitude necessary for complete flipping in time $\tau$ for a rotating field at frequency $\omega_{0}$. Thus not only is there the slight shift in the resonance frequency as calculated by Bloch and Siegert and by Stevenson, but there is also a slight change in the amplitude of the rf field necessary for complete flipping. Yet the rotating field answers are correct to within 1\% for this case. This slight difference in off amplitude makes the integral

$$
\int_{0}^{\tau} \omega_{1}(t) \mathrm{d} t=3.1440
$$

rather than $\pi$ for maximum flipping ratio. The effect of a variation in $\mathrm{rf}$ field amplitude over the path through the coil was investigated. The variation was

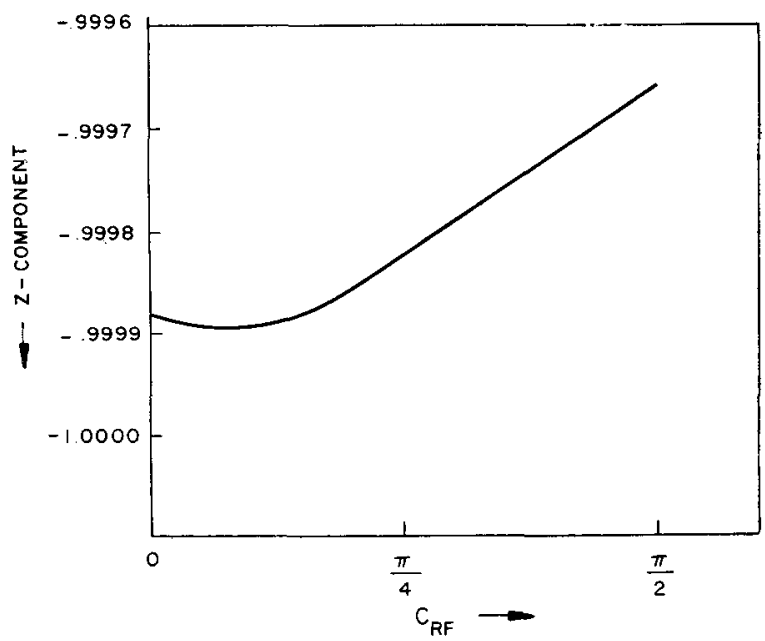

Fig. 4. $Z$ component of the neutron spin at time $\tau$ vs $C_{\mathrm{rf}}$ the amplitude of the sinusoidal spatial variation of $\mathrm{rf}$ field. The spatial variation is given by eq. (20). Eq. (19) is satisfied for each value of $C_{\text {rf }}$. The frequency is held constant at the value given in eq. (18a).
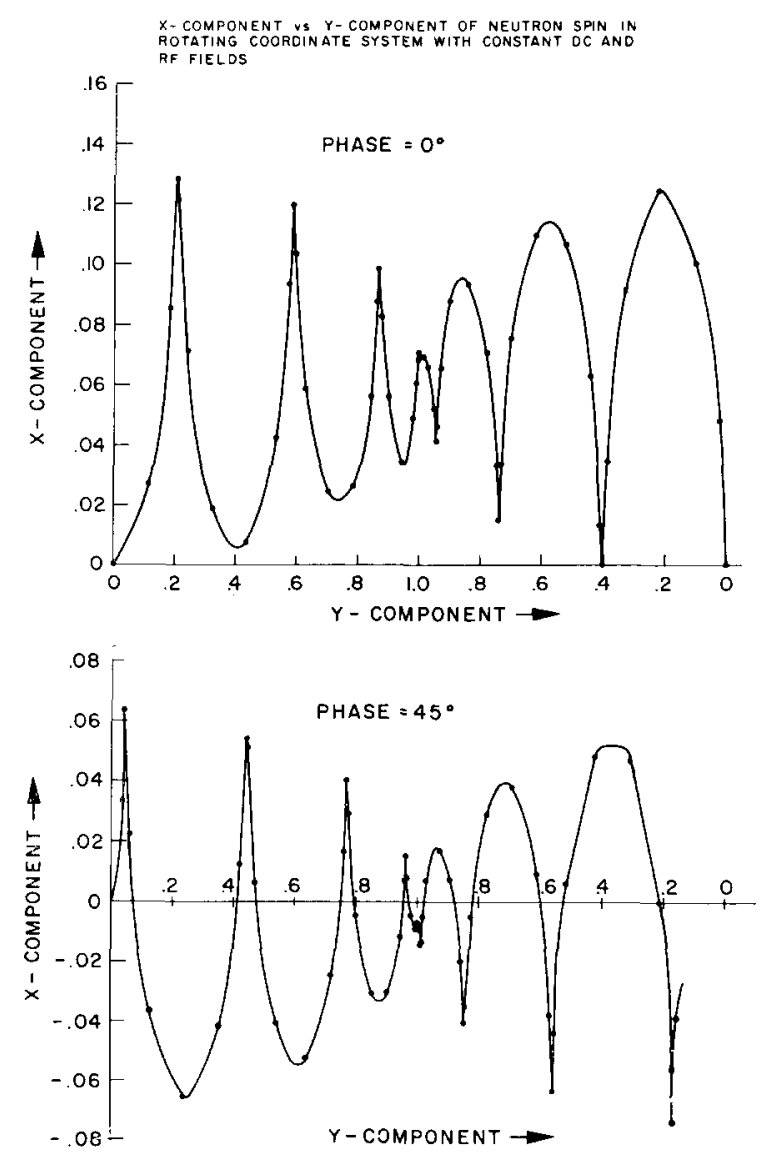

Fig. 5. The effect of phase on the projection of the neutron spin in the $x-y$ plane in the rotating coordinate system. (a) phase $=0$, (b) phase $=\pi / 4$. The points are at equal time intervals. For phase $=0$ the last point at $x=0, y=0$ is at the time the neutron leaves the coil. For phase $\pi / 4$ the last point is for $y \approx 0.2$ and $x \approx-0.04$. Apparently if the coil were a little wider both $x$ and $y$ would come close to 0 .

chosen so that eq. (19) was always satisfied. For a variation in which the amplitude of the rf field is given by

$$
B_{1}(x)=B_{10}\left(1+C_{\mathrm{rf}} \sin \pi x / L\right), \quad(0<x<L),
$$

the dependence of $M_{z}(\tau)$ on $C_{\mathrm{rf}}$ is given in fig. 4. From the viewpoint of reversing the polarization of a neutron beam, there is negligible difference between the oscillating field case shown here and the rotating field case where there is no effect from a spatial variation of the rf amplitude.

The effect of phase is more noticeable. The above flipping conditions were maximized for a neutron entering the coil when the rf was at a maximum. Keeping the values of $B_{1}$ and $\omega$ fixed while changing the phase from 0 to $\frac{1}{4} \pi$ changes $M_{z}(\tau)$ from -0.9999 to -0.9869 . Fig. 5 shows the $x$-component vs $y$-com- 
ponent in the rotating coordinate system for two choices of phase. The frequency and amplitude of the oscillating rf field are chosen as above to make the $x$ - and $y$-components return to zero for $\tau$ equal to the passage time of the $1.05 \AA$ neutron through the $2.80 \mathrm{~cm}$ coil when the neutron enters in phase with the rf field. The $y$ scale is unfolded for clarity. Note that for the phase $\varphi=\frac{1}{4} \pi$ the $x$ - and $y$-components are not zero at the last point. The points on the curves are taken at equal time intervals and the last point is at the transit time for the neutron. One can see that if the neutron spent more time in the coil for $\varphi=\frac{1}{4} \pi$ the $x$ - and $y$ components would both come toward zero. Thus for $a$ fixed rf power one needs a wider coil or for a fixed coil one needs more rf power to flip neutrons entering the coil out of phase with the $r f$. There is also the implication that the rf frequency need be changed only very slightly, if at all.

\subsection{PNPI EXPERIMENTS}

As explained in the introduction the PNPI has two sources of dc field. One is a set of alnico magnets attached to a pair of parallel steel plates shown in fig. 6 . This field is mechanically adjusted using micrometer driven shunts to give resonance for a fixed rf frequency. The other source is the current in a rectangular coil of aluminum oxide coated aluminum wire. This coil has its axis vertical. The purpose of this coil is to apply pulses of field which will destroy the resonance condition. But in the experiments reported here it was used to vary the de field. The $\mathrm{rf}$ coil is inside the de coil. A view of the coils inside the copper shield (the lid is removed) is seen in fig. 7. The neutrons enter the box through aluminum windows. The de coil is labelled B. The rf coil is just visible through the hole in the teflon spacer. Cooling air is circulated; it enters at the nozzle (N) and is directed at the coils by the use of a manifold

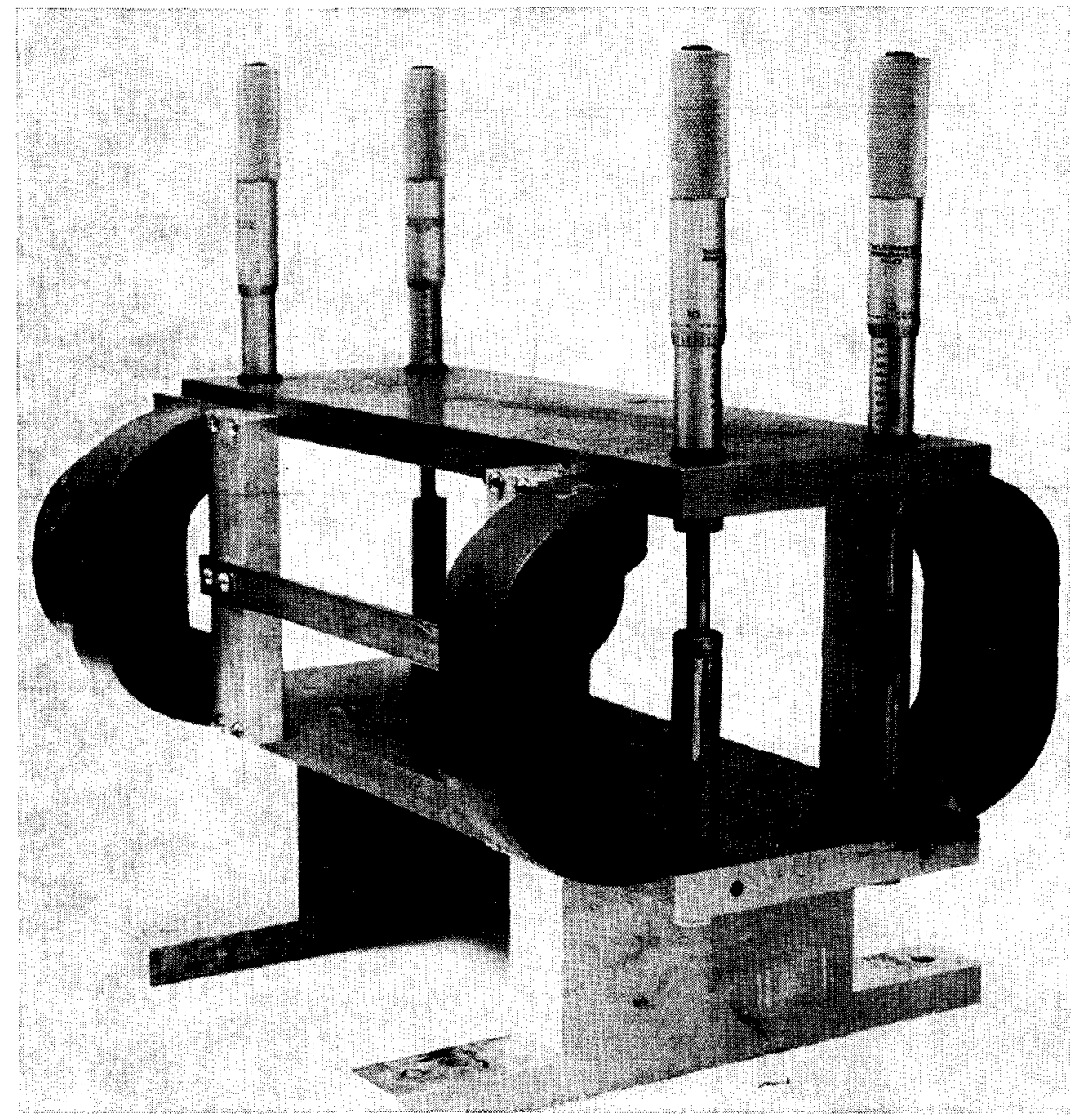

Fig. 6. Adjustable dc field for pulsed neutron polarization inverter (PNPI). The micrometers move magnetic shunts. 


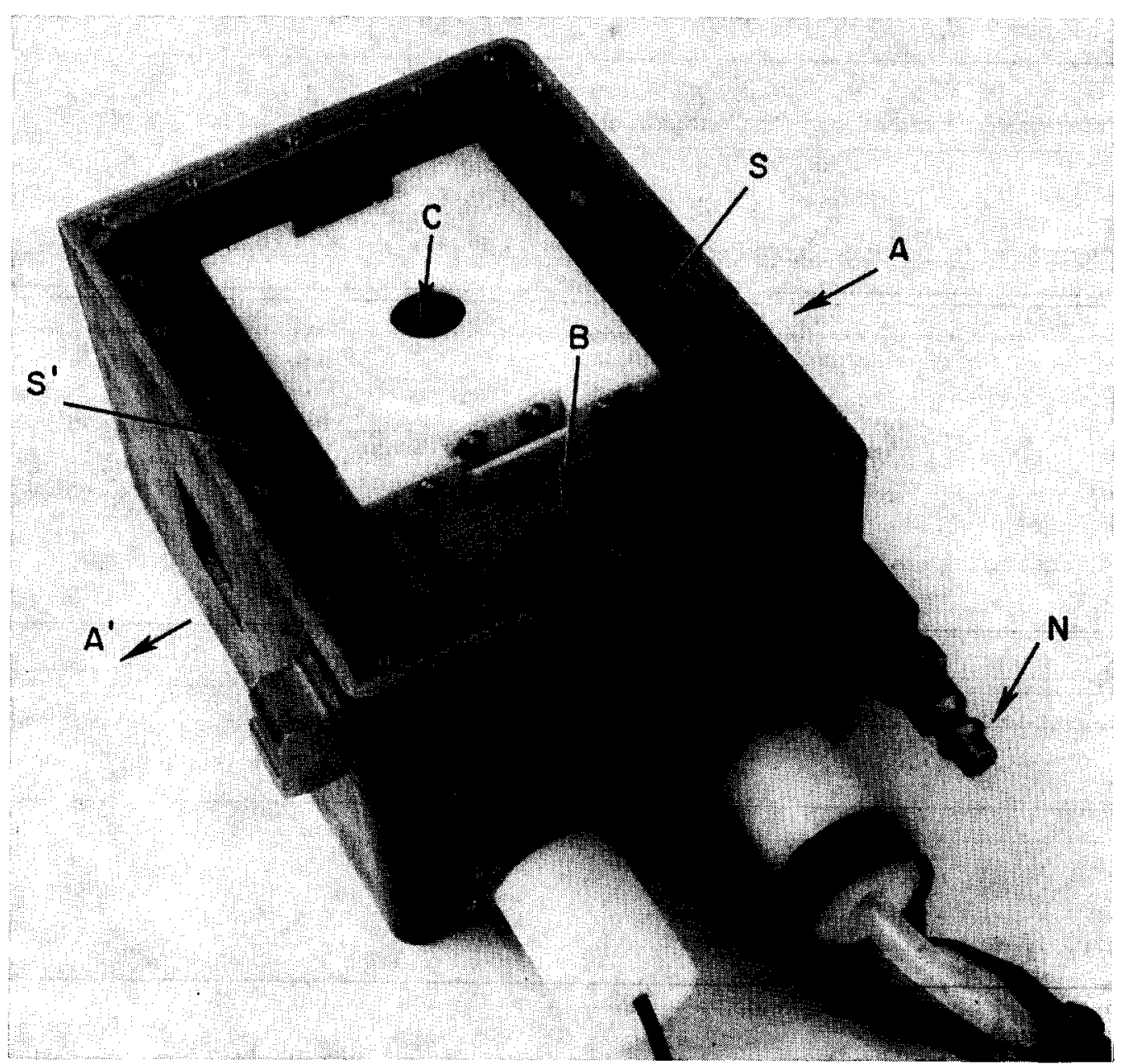

Fig. 7. Pulsed neutron polarization inverter with cover removed from the copper box. $\mathbf{A}$ and $\mathbf{A}^{\prime}$ are the thin aluminum entrance and exit ports. $B$ is the vertical de field coil. $C$ indicates the barely visible horizontal if coil. $S$ and $S^{\prime}$ are copper shields to keep the back field of the rf coil away from the neutron beum. $\mathrm{N}$ is a nozzle for air at 2 atmosphere.

and a series of nozzles. The use of two atmospheres of pressure not only aids in cooling but also in reducing discharge. As the coils are short in this design we reduce the effects of the return paths of flux by shielding with copper (S) around the beam. As the copper shield stops short of the dc coil on both sides of the box there is more of a return path through the beam for the field of the rf coil than for the field of the dc coil. The effect of this "back" field is to increase the rf amplitude necessary for resonance from $47 \mathrm{G}$ as calculated for a field over the $2.8 \mathrm{~cm}$ of the path within the coil and no back field. While the field in the coil causes a counterclockwise precession (which lies almost in the $y-z$ plane) the back fields cause precession in the opposite direction. Thus in the integral $\int_{0}^{\tau} \omega_{1}(t) \mathrm{d} t$, the $\omega_{1}$ is negative outside the coil and positive inside. For a rotating field the polarization goes as $\sin ^{2} \theta$ where [as discussed for eqs. (8), (9) and (11)] $\theta$ is $\frac{1}{2} \int_{0}^{\tau} \omega_{1}(t) \mathrm{d} t$. Fig. 8 shows a comparison of the measured flipping probability and $a \sin ^{2} \theta$ normalized to the slightly less than perfect flipping probability. The peak is at $56.5 \mathrm{G}$. From this we can estimate the back field. If one wished to get a better idea of the rf field distribution one could measure the flipping probability as a function of frequency. This would yield the Fourier transform of the variation of $\mathrm{rf}$ amplitude through the coil. For the square wave dependence of $\mathrm{rf}$ amplitude on distance in section 2 one gets

$$
P\left(\frac{1}{2} \pi\right)=\left[1+\left(\Delta \omega / \omega_{1}\right)^{2}\right]^{-1} \sin ^{2}\left[1+\left(\Delta \omega / \omega_{1}\right)^{2}\right]^{\frac{1}{2}} \frac{1}{2} \pi,(21)
$$

which is shown as the dashed curve of fig. 9. The ex- 


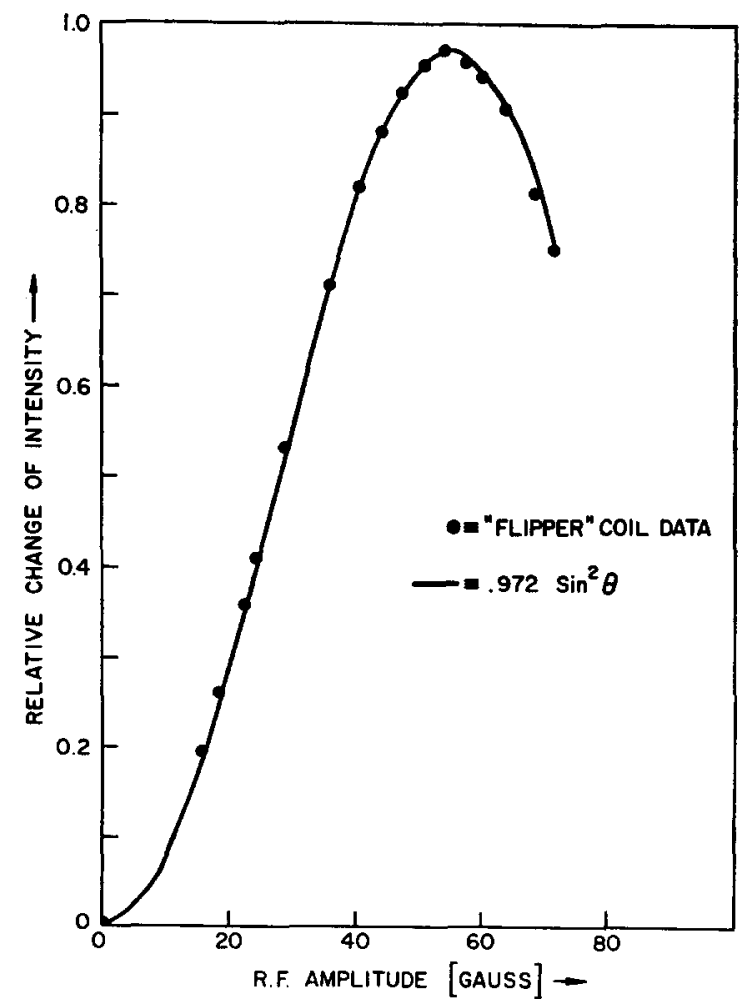

Fig. 8. The relative change of intensity of the beam from an analyser crystal vs amplitude of the rf field.

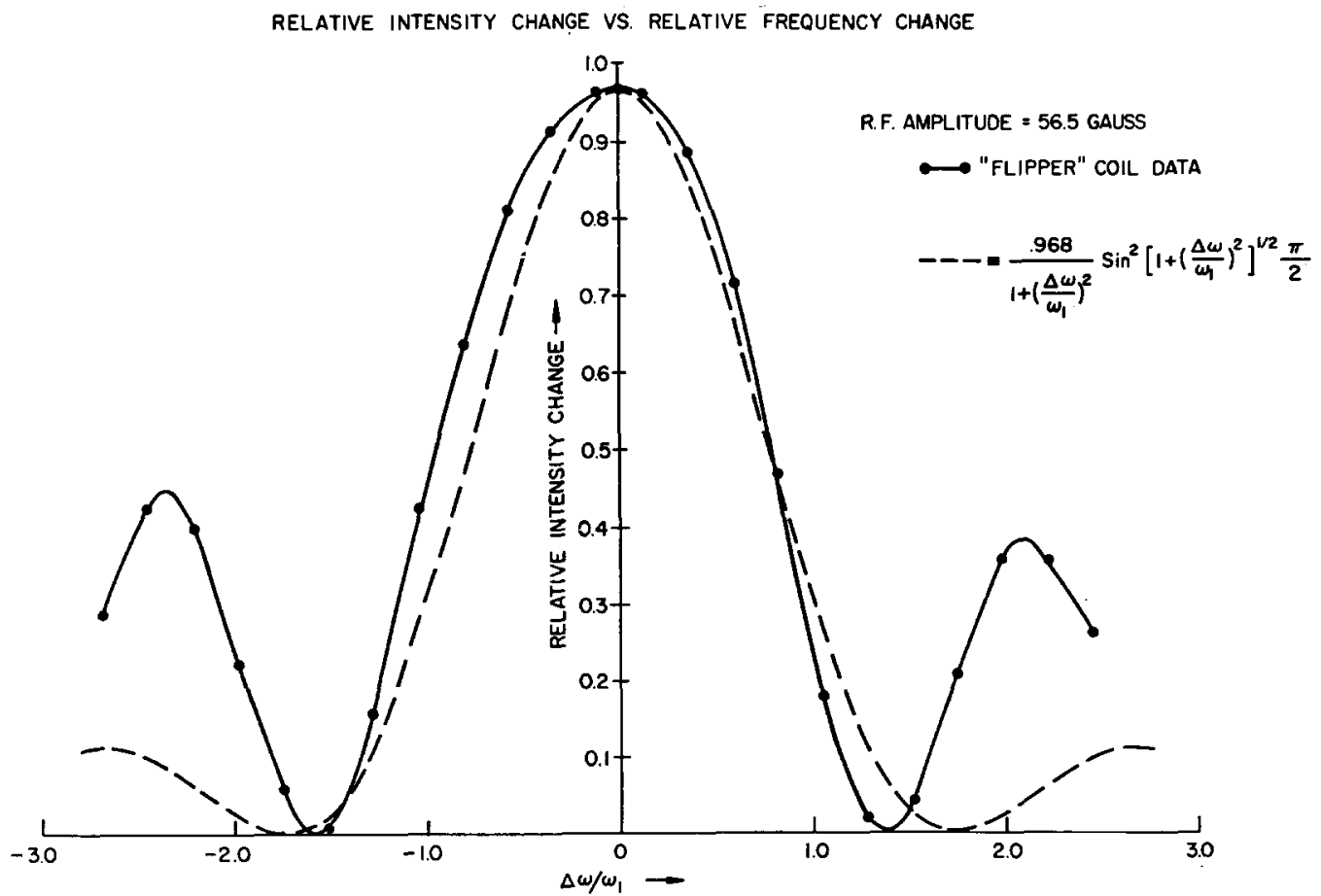

Fig. 9. The relative change of intensity of the beam from an analyser crystal vs difference of frequency and the resonance frequency, normalized to the frequency equivalent of the amplitude of the rf field $=56.5 \mathrm{G}$. The solid line is through the data points. The dotted line is calculated using eq. (21) with normalization factor of 0.968 . 
perimental points in the same figure were obtained by varying the $\mathrm{dc}$ field rather than the frequency of the $\mathrm{rf}$ field. This would be equivalent were it not for the fact that the vertical dc coil was used and it does not give a uniform field because of its short height. The result of the combination of the nonuniform dc field and the presence of the back field for the rf is to slightly decrease the field change necessary for destroying reso-

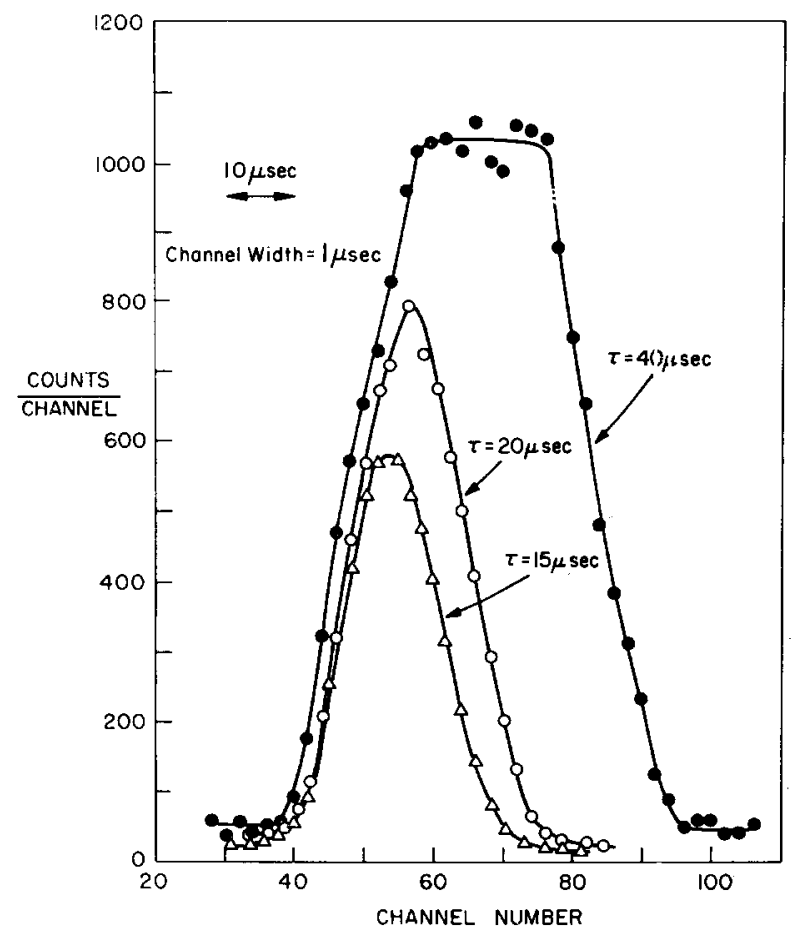

Fig. 10. Typical neutron pulses from the pulsed-neutron-polarization-inverter (PNPI). The experimental arrangement uses a $\mathrm{Co}_{0.92} \mathrm{Fe}_{0.08}$ polarizing crystal producing monoenergetic neutrons $(\lambda=1.05 \AA)$. These neutrons pass through the PNPI and the pulse of spinup neutrons are Bragg reflected by a second $\mathrm{Co}_{0.92} \mathrm{Fe}_{0.08}$ crystal. They are detected by a $\frac{1}{4} "$ dia., $40 \mathrm{~atm} .{ }^{3} \mathrm{He}$ filled detector placed approximately $1 \mathrm{~m}$ from the second crystal. The current pulse supplied to the "dc" coil has a width $\tau$ at the base and a rise (and decay) time of approximately $\frac{1}{2} \mu \mathrm{sec}$. The width of the neutron pulse $\Gamma$ is greater than $\tau$ because of the spread in incident neutron velocities and the finite width of the "dc" coil. The reason why the height of the neutron pulse decreases with decreasing $\tau$ is due in part to the fact that there is a distribution of initial positions of the neutrons in the coil at the times the pulse is turned on and off. It is found experimentally that the height of these neutron pulses of short $\Gamma$ can be increased by increasing the amplitude of the current pulse. nance. But at the same time it heightens the subsidiary maximum. This would make the accuracy requirement of the dc field setting more stringent than one would calculate from eq. (21). But in operation of the pulsed system this is not an important consideration. For one is not attempting to perfectly destroy the resonance as much as to produce a well-shaped pulse of neutrons of unflipped polarization. (The polarized neutrons are flipped during the time the pulse is off and not flipped when the resonance is destroyed.) There is a distribution of initial positions of the neutrons in the coil at the time the pulse is turned on and at the time the pulse is turned off. Also there is a finite rise time $\left(\approx \frac{1}{2} \mu \mathrm{sec}\right)$ for the pulse. The rf amplitude which produces the largest amplitude for the pulse of unflipped neutrons is considerably larger than the $40 \mathrm{G}$ which corresponds to the first minimum of fig. 9. The optimum amplitude is larger the shorter the pulse. Fig. 10 shows some typical pulses using the PNPI.

The authors wish to thank Dr. R. Nathans for stimulating this interest in pulsed-neutron-polarization-inverting. The detailed design and construction of the PNPI coils and the necessary circuitry ${ }^{8}$ ) was carried out by Mr. R. Forgacs to whom we are much indebted. Most of this paper is based on part of a thesis submitted by one of us (H.K.) to the Department of Nuclear Engineering at the University of Michigan.

\section{References}

1) For a review of the general applications of resonance methods, see N. F. Ramsey, Molecular beams (Oxford, 1956) and the references therein.

2) For a review of the specific applications to thermal neutrons, see for example R. Nathans and S. J. Pickart, in Magnetism, vol. III (eds. G. T. Rado and H. Suhl; Academic Press, 1963).

3) I. I. Rabi, N. F. Ramsey and J. Schwinger, Rev. Mod. Phys. 26 (1954) 167.

4) F. Bloch and A. Siegert, Phys. Rev. 57 (1940) 522.

5) A. F. Stevenson, Phys. Rev. 58 (1940) 1011.

6) See ref. ${ }^{1}$ ), p. 122.

$\left.{ }^{7}\right)$ The analog computer used in these investigations was the Pace Model \# 16-31R-4 made by Electronic Associates Inc. in the Nuclear Engineering Department at the University of Michigan.

8) R. Forgacs, to be published. 